\title{
BIOHEAT TRANSFER EQUATION. THE PROBLEM OF FDM EXPLICIT SCHEME STABILITY
}

\author{
Wioletta Tuzikiewicz ${ }^{1}$, Mateusz Duda ${ }^{2}$ \\ ${ }^{I}$ Institute of Mathematics, Czestochowa University of Technology \\ Częstochowa, Poland \\ ${ }^{2}$ University of Occupational Safety Management in Katowice \\ Katowice, Poland \\ wioletta.tuzikiewicz@im.pcz.pl
}

\begin{abstract}
In the paper the problem of explicit FDM scheme stability for the bio-heat transfer equation (the Pennes equation) is discussed. To formulate the appropriate condition the von Neumann approach is applied. The first chapter contains the known derivation of FDM stability condition for the Fourier equation. In the second part, a similar condition is found for the case of the bio-heat transfer equation containing both the perfusion and metabolic heat sources.
\end{abstract}

Keywords: bio-heat transfer, finite difference method, stability condition, von Neumann method

\section{Introduction}

The problem of numerical schemes stability is closely associated with a numerical error. The FDM scheme is stable when the errors made at one time step of the calculation do not cause the errors to increase as the computations are continued [1]. If, on the contrary, the errors grow with time, the numerical scheme is said to be unstable. The stability of numerical schemes can be investigated by performing von Neumann stability analysis. According to this theory, the approximation error carried by $\theta_{i, j}^{f}(2 \mathrm{D}$ problem) at every node of space $(i, j)$ and time $f$ is assumed to have a wave form with the wave numbers denoted by $s_{1}, s_{2}$ and the amplitude by $\delta$ :

$$
\theta_{i, j}^{f}=\delta^{f} \exp \left[I\left(s_{1} x_{i}+s_{2} y_{j}\right)\right], \quad I=\sqrt{-1}
$$

As time progresses, to assure convergence, the amplitude of the approximation error must be less than unity, i.e. $\left|\theta_{i, j}^{f}\right|<1[2]$. 
As an example, the well-known 2D Fourier equation in the form

$$
(x, y) \in \Omega: \quad \frac{\partial T(x, y, t)}{\partial t}=a \nabla^{2} T(x, y, t)
$$

is considered. In this equation $a=\lambda / c$ is a thermal diffusion coefficient ( $c$ is a volumetric specific heat, $\lambda$ is a thermal conductivity), $T, x, y, t$ denote the temperature, spatial co-ordinates and time.

On the external surface of the system the boundary conditions in a general form

$$
(x, y) \in \Gamma: \quad \Phi\left[T(x, y, t), \frac{\partial T(x, y, t)}{\partial n}\right]=0
$$

is given ( $\partial / \partial n$ denotes a normal derivative).

The initial condition is also given

$$
t=0: \quad T(x, 0)=T_{0}
$$

Let us consider the domain oriented in Cartesian co-ordinate system covered by the rectangular differential mesh with steps $h$ and $\mathrm{k}$. Additionally, $f-1$ and $f$ denote two successive time levels with step $\Delta t$. We introduce the local numeration and the central point of the star is denoted as $P_{i, j}$, while the adjacent nodes as $P_{i+1, j}$, $P_{i-1, j}, P_{i, j+1}$ and $\mathrm{P}_{i, j-1}$.

The FDM equation for node $P_{i, j}$ (explicit scheme) can be written in the form

$$
\frac{T_{i, j}^{f}-T_{i, j}^{f-1}}{\Delta t}=a\left(\frac{T_{i+1, j}^{f-1}-2 T_{i, j}^{f-1}+T_{i-1, j}^{f-1}}{h^{2}}+\frac{T_{i, j+1}^{f-1}-2 T_{i, j}^{f-1}+T_{i, j-1}^{f-1}}{k^{2}}\right)
$$

or

$$
T_{i}^{f}=\left(1-\frac{2 a \Delta t}{h^{2}}-\frac{2 a \Delta t}{k^{2}}\right) T_{i}^{f-1}+\frac{a \Delta t}{h^{2}}\left(T_{i+1, j}^{f-1}+T_{i-1, j}^{f-1}\right)+\frac{a \Delta t}{k^{2}}\left(T_{i, j+1}^{f-1}+T_{i, j-1}^{f-1}\right)
$$

Now, the formula (1) will be applied and then

$$
\begin{aligned}
& \delta^{f} \exp \left[I\left(s_{1} x_{i}+s_{2} y_{j}\right)\right]=\left(1-\frac{2 a \Delta t}{h^{2}}-\frac{2 a \Delta t}{k^{2}}\right) \delta^{f-1} \exp \left[I\left(s_{1} x_{i}+s_{2} y_{j}\right)\right]+ \\
& \frac{a \Delta t}{h^{2}} \delta^{f-1}\left\{\exp \left[I\left(s_{1} x_{i+1}+s_{2} y_{j}\right)\right]+\exp \left[I\left(s_{1} x_{i-1}+s_{2} y_{j}\right)\right]\right\}+ \\
& \frac{a \Delta t}{k^{2}} \delta^{f-1}\left\{\exp \left[I\left(s_{1} x_{i}+s_{2} y_{j+1}\right)\right]+\exp \left[I\left(s_{1} x_{i}+s_{2} y_{j-1}\right)\right]\right\}
\end{aligned}
$$


or dividing by $\delta^{f-1} \exp \left[I\left(s_{1} x_{i}+s_{2} y_{j}\right)\right]$

$$
\begin{aligned}
& \delta=\left(1-\frac{2 a \Delta t}{h^{2}}-\frac{2 a \Delta t}{k^{2}}\right)+\frac{a \Delta t}{h^{2}}\left[\exp \left(I s_{1} h\right)+\exp \left(-I s_{1} h\right)\right]+ \\
& \frac{a \Delta t}{k^{2}}\left[\exp \left(I s_{2} k\right)+\exp \left(-I s_{2} k\right)\right]
\end{aligned}
$$

Using the Euler formula one can be written

$$
\delta=\left(1-\frac{2 a \Delta t}{h^{2}}-\frac{2 a \Delta t}{k^{2}}\right)+\frac{2 a \Delta t}{h^{2}} \cos \left(s_{1} h\right)+\frac{2 a \Delta t}{k^{2}} \cos \left(s_{2} k\right)
$$

and next

$$
\delta=1-\frac{2 a \Delta t}{h^{2}}\left[1-\cos \left(s_{1} h\right)\right]-\frac{2 a \Delta t}{k^{2}}\left[1-\cos \left(s_{2} k\right)\right]
$$

Because $1-\cos \alpha=2 \sin ^{2}\left(\frac{\alpha}{2}\right)$, therefore

$$
\delta=1-\frac{4 a \Delta t}{h^{2}} \sin ^{2} \frac{s_{1} h}{2}-\frac{4 a \Delta t}{k^{2}} \sin ^{2} \frac{s_{2} k}{2}
$$

The condition $\left|\theta_{i, j}^{f}\right|<1$ leads to the system of inequalities

$$
\frac{4 a \Delta t}{h^{2}} \sin ^{2} \frac{s_{1} h}{2}+\frac{4 a \Delta t}{k^{2}} \sin ^{2} \frac{s_{2} k}{2}>0
$$

and

$$
1-\frac{2 a \Delta t}{h^{2}} \sin ^{2} \frac{s_{1} h}{2}-\frac{2 a \Delta t}{k^{2}} \sin ^{2} \frac{s_{2} k}{2}>0
$$

The first of them is the unconditional inequality, while the worst situation in the case of the second inequality takes place when $\sin ^{2} \frac{s_{1} h}{2}=1, \sin ^{2} \frac{s_{2} k}{2}=1$ and then

$$
1-\frac{2 a \Delta t}{h^{2}}-\frac{2 a \Delta t}{k^{2}}>0
$$

In this way the well-known stability condition for the linear parabolic equations is found. The physical interpretation of the last formula can be found in [3]. 


\section{Bio-heat transfer equation}

Thermal processes proceeding in the domain of living tissue are, as a rule, described by the following partial differential equation (2D problem) - e.g. [4-6]

$$
(x, y) \in \Omega: \quad c \frac{\partial T(x, y, t)}{\partial t}=\lambda \nabla^{2} T(x, y, t)+G_{B} C_{B}\left[T_{B}-T(x, y, t)\right]+Q_{m e t}
$$

As previously explained, $c$ is the mean volumetric specific heat of the skin tissue, $\lambda$ is the mean thermal conductivity. Additionally, $G_{B}\left[\mathrm{~m}^{3}\right.$ blood $/ \mathrm{m}^{3}$ tissue $\left.\cdot \mathrm{s}\right]$ is the perfusion coefficient, $c_{B}$ is the volumetric specific heat of blood, $Q_{m e t}$ is the metabolic heat source. It should be pointed out that the second component on the right-hand side of equation (15) corresponds to the so-called perfusion heat source and it is connected with the presence of the big number of capillaries in the domain of soft tissue. The equation (15) is supplemented by the appropriate boundary and initial conditions.

The FDM equation for the node $(i, j)$ written in the explicit form is the following

$$
\begin{aligned}
& c \frac{T_{i, j}^{f}-T_{i, j}^{f-1}}{\Delta t}=\lambda\left(\frac{T_{i+1, j}^{f-1}-2 T_{i, j}^{f-1}+T_{i-1, j}^{f-1}}{h^{2}}+\frac{T_{i, j+1}^{f-1}-2 T_{i, j}^{f-1}+T_{i, j-1}^{f-1}}{k^{2}}\right)+ \\
& G_{B} c_{B}\left(T_{B}-T_{i, j}^{f-1}\right)+Q_{m e t}
\end{aligned}
$$

After simple mathematical manipulations one obtains

$$
\begin{aligned}
& T_{i}^{f}=\left(1-\frac{2 \lambda \Delta t}{c h^{2}}-\frac{2 \lambda \Delta t}{c k^{2}}-\frac{G_{B} c_{B} \Delta t}{c}\right) T_{i}^{f-1}+\frac{\lambda \Delta t}{c h^{2}}\left(T_{i+1, j}^{f-1}+T_{i-1, j}^{f-1}\right)+ \\
& \frac{\lambda \Delta t}{c k^{2}}\left(T_{i, j+1}^{f-1}+T_{i, j-1}^{f-1}\right)+q
\end{aligned}
$$

where

$$
q=\left(G_{B} c_{B} T_{B}+Q_{m e t}\right) \frac{\Delta t}{c}
$$

As previously explained, the formula (1) is applied and then

$$
\begin{aligned}
& \delta^{f} \exp \left[I\left(s_{1} x_{i}+s_{2} y_{j}\right)\right]= \\
& \left(1-\frac{2 \lambda \Delta t}{c h^{2}}-\frac{2 \lambda \Delta t}{c k^{2}}-\frac{G_{B} c_{B} \Delta t}{c}\right) \delta^{f-1} \exp \left[I\left(s_{1} x_{i}+s_{2} y_{j}\right)\right]+ \\
& \frac{\lambda \Delta t}{h^{2}} \delta^{f-1}\left\{\exp \left[I\left(s_{1} x_{i+1}+s_{2} y_{j}\right)\right]+\exp \left[I\left(s_{1} x_{i-1}+s_{2} y_{j}\right)\right]\right\}+ \\
& \frac{\lambda \Delta t}{k^{2}} \delta^{f-1}\left\{\exp \left[I\left(s_{1} x_{i}+s_{2} y_{j+1}\right)\right]+\exp \left[I\left(s_{1} x_{i}+s_{2} y_{j-1}\right)\right]\right\}
\end{aligned}
$$


or

$$
\begin{aligned}
& \delta=\left(1-\frac{2 \lambda \Delta t}{c h^{2}}-\frac{2 \lambda \Delta t}{c k^{2}}-\frac{G_{B} c_{B} \Delta t}{c}\right)+\frac{\lambda \Delta t}{c h^{2}}\left[\exp \left(I s_{1} h\right)+\exp \left(-I s_{1} h\right)\right]+ \\
& \frac{\lambda \Delta t}{c k^{2}}\left[\exp \left(I s_{2} k\right)+\exp \left(-I s_{2} k\right)\right]
\end{aligned}
$$

One can see that the component $q$ is omitted because the constant value in the FDM equation does not affect the stability of the explicit scheme.

Proceeding in a similar way as in the case of the Fourier equation one finally obtains

$$
1-\frac{2 \lambda \Delta t}{c h^{2}}-\frac{2 \lambda \Delta t}{c k^{2}}-\frac{G_{B} c_{B} \Delta t}{c}>0
$$

or

$$
\Delta t<\frac{c}{\frac{2 \lambda}{h^{2}}+\frac{2 \lambda}{k^{2}}+G_{B} c_{B}}
$$

The critical time step is equal to

$$
\Delta t_{c r}=\frac{c}{\frac{2 \lambda}{h^{2}}+\frac{2 \lambda}{k^{2}}+G_{B} c_{B}}
$$

The adoption of a larger time step leads to the instability and the results obtained are physically incorrect.

As an example, the following input data are taken into account: volumetric specific heat of tissue $c=3 \mathrm{MJ} /\left(\mathrm{m}^{3} \mathrm{~K}\right)$, thermal conductivity of tissue $\lambda=0.3 \mathrm{~W} /(\mathrm{mK})$, volumetric specific heat of blood $c_{B}=4 \mathrm{MJ} /\left(\mathrm{m}^{3} \mathrm{~K}\right)$, perfusion coefficient $G_{B}=0.0005$ and then assuming $h=k=0.001 \mathrm{~m}$ one obtains the critical time step equal to $2.5 \mathrm{~s}$, for $h=k=0.005 \mathrm{~m}: \Delta t_{c r}=0.625 \mathrm{~s}$, while for $h=0.001 \mathrm{~m}, k=0.0005 \mathrm{~m}: \Delta t_{c r}=1 \mathrm{~s}$.

The mesh steps considered above are the typical ones in the numerical modelling of bio-heat transfer problems using the finite difference method.

\section{Conclusions}

The von Neumann method is used as a tool of the stability condition formulation for the case of the bio-heat transfer equation and the explicit FDM scheme application (at the stage of computations). The authors confirmed the fact that the accompanying temperature coefficient in the perfusion heat source must be taken into account in the formula determining the critical time step. 


\section{Acknowledgement}

This paper is a part of the Project PB3/2013 sponsored by WSZOP Katowice.

\section{References}

[1] Tzou D.Y., Macro- to Microscale Heat Transfer: The Lagging Behavior, John Wiley \& Sons Ltd., 2015.

[2] Anderson J.D., Computational Fluid Dynamics. The Basics with Applications, McGraw Hill, 1994.

[3] Mochnacki B., Suchy J.S., Numerical Methods in Computations of Foundry Processes, PFTA, Cracow 1995.

[4] Mochnacki B., Majchrzak E., Duda M., Numerical modeling of thermal processes in the living tissue domain secured with a layer of protective clothing, Journal of Applied Mathematics and Computational Mechanics 2014, 13, 1, 97-102.

[5] Mochnacki B., Majchrzak E., Duda M., Simplified model of thermal interactions between environment, protective clothing and skin tissue, Journal of Applied Mathematics and Computational Mechanics 2014, 13, 4, 93-96.

[6] Ciesielski M., Mochnacki B., Application of the control volume method using the Voronoi polygons for numerical modeling of bio-heat transfer processes, Journal of Theoretical and Applied Mechanics 2014, 52, 4, 927-935. 